\title{
Modified continuity equation using left ventricular outflow tract three-dimensional imaging for aortic valve area estimation
}

\author{
Pedro Pinto Teixeira MD ${ }^{1}$ (D) | Ruben Ramos MD $^{1}$ | Pedro Rio MD ${ }^{1}$ | Luísa Moura Branco \\ $M^{1}$ | Guilherme Portugal MD ${ }^{1}$ | Ana Abreu MD ${ }^{1}$ | Ana Galrinho $M^{1}$ | Hugo Marques \\ $M^{2}$ | Luísa Figueiredo $M D^{2} \mid$ Rui Cruz Ferreira $M^{1}$
}

${ }^{1}$ Cardiology Department, Hospital de Santa Marta, Lisboa, Portugal

${ }^{2}$ Radiology Department, Hospital de Santa Marta, Lisboa, Portugal

Correspondence

Pedro Pinto Teixeira, Cardiology Department, Hospital de Santa Marta, Lisboa, Portugal.

Email: phpintoteixeira@gmail.com
Purpose: Aortic valve area (AVA) is usually estimated by the continuity equation (CE) in which the left ventricular outflow tract (LVOT) area is calculated assuming a circular shape. This study aimed to compare measurements of LVOT area using standard 2D transthoracic echocardiography (2DTTE), 3D transesophageal echocardiography (3DTEE), and multidetector computed tomography (MDCT) and assess their relative impact on AVA estimated by the CE.

Methods and Results: We prospectively enrolled 60 patients with severe aortic stenosis (AS) referred for transcatheter aortic valve replacement (TAVR) who systematically underwent 2DTTE, 3DTEE, and MDCT. Mean LVOT areas obtained by 2DTTE $\left(3.28 \pm 0.66 \mathrm{~cm}^{2}\right)$ and 3DTEE $\left(3.95 \pm 0.90 \mathrm{~cm}^{2}\right)$ were significantly underestimated when compared to the mean MDCT LVOT area $\left(4.31 \pm 0.99 \mathrm{~cm}^{2}\right)$. LVOT was rather elliptical than round, with a mean eccentricity index of 1.47 (ratio of maximum to minimum LVOT diameters) assessed by MDCT. Mean TTE AVA estimated by the CE was $0.62 \pm 0.20 \mathrm{~cm}^{2}$. Substitution of 2DTTE LVOT area by 3DTEE LVOT area in the CE resulted in AVA of $0.74 \pm 0.24 \mathrm{~cm}^{2}$, while using MDCT LVOT area held an AVA of $0.80 \pm 0.24 \mathrm{~cm}^{2}$. MDCT-derived AVA was similar to MDCT planimetric AVA and allowed $24 \%$ of patients to be reclassified from severe to moderate AS.

Conclusions: 2DTTE and 3DTEE underestimate LVOT area when compared to MDCT with significant impact on AVA estimation. Assessment through MDCT fusion AVA may be of incremental value in patients with discrepant severity criteria for AS.

\section{KEYWORDS}

aortic stenosis, aortic valve replacement, computed tomography, three-dimensional echocardiography

\section{1 | INTRODUCTION}

Accurate estimation of aortic valve area (AVA) is crucial to identify patients with severe aortic stenosis (AS) who might be eligible for aortic valve replacement treatment. ${ }^{1-3}$ The measurement of AVA using two-dimensional (2D) transthoracic echocardiography (TTE) is based on the widely accepted continuity equation, which assumes a circular geometry of the left ventricular outflow tract (LVOT). ${ }^{1-5}$ However, preceding studies using three-dimensional (3D) imaging methods such as multidetector computed tomography (MDCT), magnetic resonance 
imaging, or 3D echocardiography have shown the LVOT shape is often elliptical, leading to a potential underestimation of LVOT area and AVA by 2DTTE. ${ }^{6-12}$ In addition to AVA estimation, other echocardiographic criteria are taken into consideration to assess the AS severity such as transaortic gradients, dimensionless index, and planimetry AVA. ${ }^{1-3}$ However, there are often discrepancies between these criteria and continuity equation 2DTTE-derived AVA, raising concerns about the accuracy of AVA estimation through this method. ${ }^{11,13}$

Multidetector computed tomography and 3D transesophageal echocardiography (3DTEE) are already routinely used in the preprocedural assessment of the aortic valve root and apparatus in the setting of transcatheter aortic valve replacement (TAVR). ${ }^{14,15}$ Moreover, these 3D imaging techniques allow direct LVOT area measurement by planimetry with high spatial resolution and image quality, which could then be introduced into the continuity equation for AVA calculation, avoiding the error associated with 2DTTE-derived LVOT area estimation. ${ }^{16,17}$ The incremental value of 3DTEE and MDCT with regard to LVOT and AVA evaluation is still scarcely explored in the literature.

The aim of this study was to (i) compare measurements of LVOT area acquired by 2DTTE, 3DTEE, and MDCT in patients with AS being assessed for TAVR; (ii) assess the impact of a modified multimodality continuity equation incorporating either 3DTEE or MDCT-derived LVOT areas; (iii) evaluate the congruence between 3D-derived AVA and various echocardiographic severity criteria for AS.

\section{2 | METHODS}

From April 2014 to December 2015, we prospectively enrolled 60 patients with severe aortic stenosis (defined by 2DTTE estimated AVA $<0.6 \mathrm{~cm}^{2} / \mathrm{m}^{2}$ using the continuity equation) who were referred for TAVR assessment. Patients were included if they had performed 2DTTE, 3DTEE, and MDCT at our institution within 1 week of each other. Patients with previous surgery of the aortic valve or ascending aorta were excluded. The study protocol was approved by the local ethics committee, with waiver of individual informed consent.

\section{1 | Echocardiography}

Echocardiography studies were executed using a commercially available echocardiographic system (iE33 Philips Medical Systems, Andover, MA, USA) using an S5-1 array probe for 2D echocardiography. All measurements were performed according to recommendations of the American Society of Echocardiography. ${ }^{1}$ From the apical long-axis or five-chamber views, continuous-wave (CW) Doppler spectral recordings were collected, enabling assessment of peak and mean transaortic gradients, as well as of velocity-time integrals (VTI) across the aortic valve. ${ }^{1}$ The highest aortic valve velocity was systematically sought in all patients and was found in nonapical locations (right parasternal or suprasternal) in nine patients. LVOT area was derived in a standard fashion from the LVOT sagittal diameter [(LVOT diameter/2 $)^{2} \times \Pi$ ], obtained on a zoomed parasternal long-axis view, $5 \mathrm{~mm}$ below the aortic annulus, in mid-systole. ${ }^{1}$ The VTI of the LVOT was assessed by spectral pulsed-wave (PW) Doppler with the sample volume positioned at the same level of the LVOT diameter measurement. ${ }^{1}$ Two-dimensional transthoracic AVA (2DTTE AVA) was calculated using the continuity equation in accordance with the current guidelines: (LVOT area $x$ LVOT $_{V T I}$ )/AV ${ }_{V T 1}$, where LVOT ${ }_{V T I}$ is the velocity-time integral of LVOT flow, and $A V_{V T I}$ is the velocity-time integral of aortic valve flow. ${ }^{1-3}$

3DTEE was performed with the same echocardiographic system using a X7-2t xMATRIX probe. The examination was undertaken with minimal patient sedation. From the mid-esophageal position, real time $3 \mathrm{D}$ imaging of a pyramidal volume $\left(60^{\circ} \times 30^{\circ}\right)$ of the aortic valve and LVOT was obtained. Settings were optimized using narrow-angled acquisition mode to ensure frame rates around $25 \mathrm{~Hz}$. A total of three different cycles were recorded in each patient. All volumetric images were analyzed offline by trained readers using a commercially available software package (3D Q-Lab, Philips Medical Systems). After choosing the mid-systolic frame, from the long-axis aortic view, perpendicular 2D planes were shifted and rotated to find the cross-sectional LVOT $5 \mathrm{~mm}$ below the aortic annulus, where LVOT area was planimetered (Figure 1). LVOT minimum and maximum diameters were also measured at this level to calculate the eccentricity index (maximum LVOT diameter/minimum LVOT diameter). The eccentricity index values ranged from 1 to 2, with a value of 1 indicating a perfect circle and greater values indicating a progressively more elliptical shape.

Intra-observer variability and inter-observer variability for LVOT diameter on 2DTTE and for LVOT area measurement on 3DTEE were assessed using a randomly selected subset of 15 patients. Measurements were repeated by the same observer after an interval $\geq 1$-month and by a second independent blinded reader. Reproducibility was estimated by intra-class correlation coefficient, with good agreement defined as $>0.80$.

\subsection{Multidetector computed tomography (MDCT)}

Multidetector computed tomography scans were performed using a 64-detector scanner (VCT Lightspeed, GE Healthcare, Milwaukee, WI, USA) after administration of iodinated contrast agent $(90-110 \mathrm{~mL}$ of Ultravist 370 ) at $4-5 \mathrm{~mL} / \mathrm{s}$ followed by $30-50 \mathrm{~mL}$ of normal saline at the same rate. Data were acquired using a retrospective ECGcontrolled tube current modulation technique, where the highest tube current (450-500 mA) was applied only during the systolic phase of the cardiac cycle. Images were reconstructed from phase $20 \%$ to $50 \%$ of the RR interval, with $5 \%$ interval increments. Mean radiation dose for the entire protocol (which comprehends thoracic and abdominal scan for vascular access study for TAVR) was $19 \pm 5 \mathrm{mSv}$.

Multidetector computed tomography images were analyzed on a dedicated CT workstation (iNtuition, TeraRecon, San Mateo, CA, USA) by an experienced cardiac CT reader who was blinded to all echocardiographic data. The largest cross-sectional area of the LVOT was measured manually at mid-systole $(20 \%-40 \%$ of the RR interval depending on the heart rate) just below the level of the aortic valve "hinge points" and using double oblique images to identify the true short-axis (Figure 2). ${ }^{17}$ LVOT minimum and maximum diameters were also collected at this level for determination of the eccentricity index. 

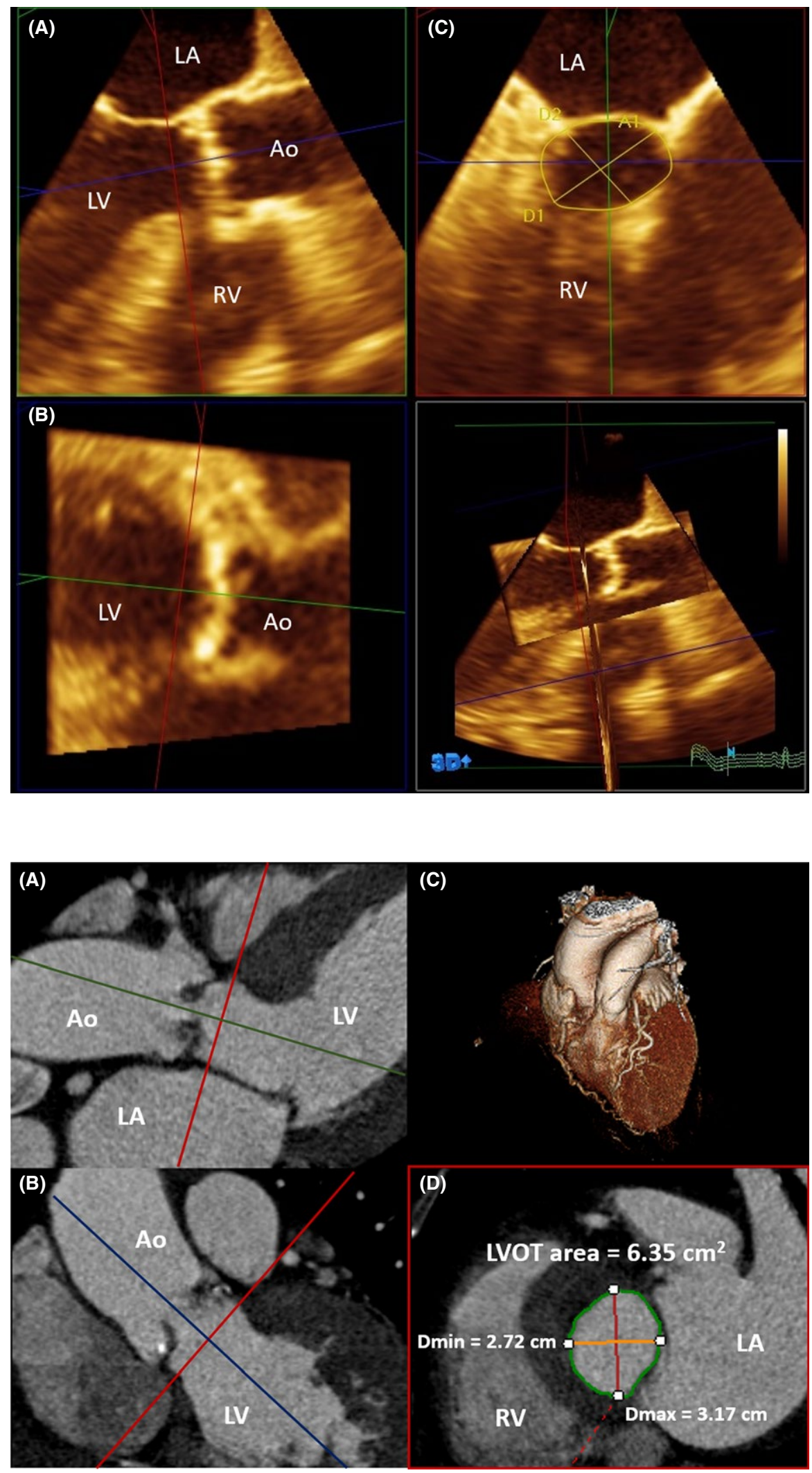

FIGURE 1 Measurement of LVOT area and minimum and maximum LVOT diameters using 3D transesophageal echocardiography. A. Parasternal longaxis plane. B. Transverse plane. C. LVOT short-axis plane. LVOT area (A1) was $6.2 \mathrm{~cm}^{2}$. LVOT maximum diameter (D1) was $3.24 \mathrm{~cm}$, and LVOT minimum diameter (D2) was $2.60 \mathrm{~cm}$. LA=left atrium; LV=left ventricle; $A o=$ ascending aorta; $R V=$ right ventricle
FIGURE 2 Measurement of the left ventricular outflow tract (LVOT) area and minimum (Dmin) and maximum (Dmax) diameters using multidetector computed tomography. The LVOT short-axis view (D) is obtained from two orthogonal views ( $A$ and $B$ ). Three-dimensional whole heart is depicted in (C). $L A=$ left atrium; $L V=$ left ventricle; $A o=$ ascending aorta; $\mathrm{RV}=$ right ventricle
Planimetry AVA was assessed manually at maximal aortic valve opening by scrolling through the short-axis images toward the tip of the cusps until the smallest orifice was found. AVA was then traced at the inside borders of the coronary cusps (Figure 3). ${ }^{17,18}$
Intra-observer variability and inter-observer variability for LVOT area quantification on MDCT were evaluated using a random subset of 15 patients, with measurements repeated by the same observer after an interval $\geq 1$-month and by a second independent blinded reader. 
FIGURE 3 Measurement of AVA by planimetry using MDCT. AVA $\left(0.98 \mathrm{~cm}^{2}\right)$ was measured in mid-systole at the tip of the aortic valve leaflets. Short-axis view (C) of the aortic valve is obtained from 2 orthogonal views ( $A$ and $B$ ). LA=left atrium; $\mathrm{LV}=$ left ventricle; $\mathrm{Ao}=$ ascending aorta

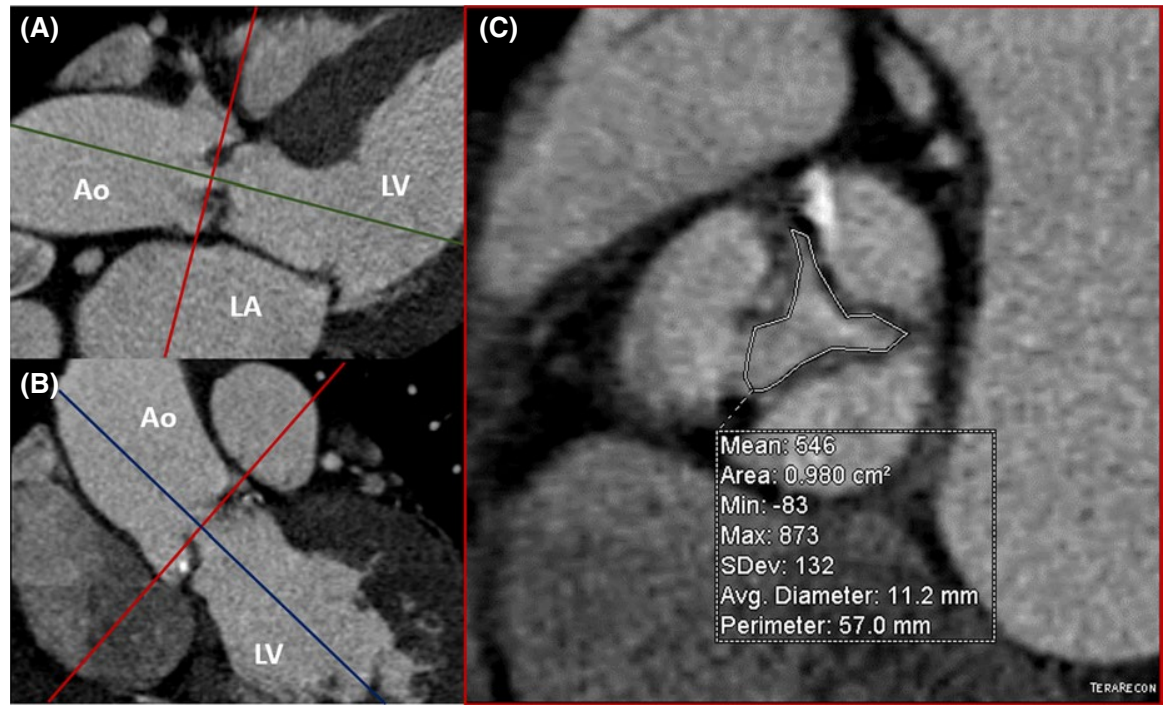

Reproducibility was estimated by intra-class correlation coefficient, with good agreement defined as $>0.80$.

\section{3 | Modified continuity equation for AVA estimation}

We replaced the conventional 2DTTE LVOT area in the continuity equation by either the 3DTEE LVOT area or the MDCT LVOT area to generate a modified multimodality continuity equation as follows: (3DTEE LVOT area or MDCT LVOT area x LVOT VTI)/Aortic valve VTI. Pulsed-wave and continuous-wave VTI through the LVOT and aortic valve were constant and assessed in a standard fashion by 2DTTE. AVAs estimated through this modified continuity equation were labeled as 3DTEE fusion AVA and MDCT fusion AVA.

\subsection{Statistical analysis}

Continuous variables are presented as mean value \pm standard deviation and categorical variables as absolute numbers and percentages. LVOT and AVA data were compared using two-sided paired $t$ test, after checking normal distribution of data and homogeneity of variances. Pearson correlation coefficient was used to test associations between continuous variables. Bland-Altmann analysis was performed to systematically assess the differences between the LVOT areas measured by 2DTTE, 3DTEE, and MDCT. ${ }^{19}$ A probability value of $<.05$ was accepted as statistically significant. Statistical analysis was performed using SPSS Statistics, version 21.

\section{3 | RESULTS}

Of the 60 consecutive patients enrolled, five were excluded due to inadequate echocardiographic or tomographic images and three due to concomitant conditions that rendered invalid the continuity equation (two patients with significant aortic regurgitation and one patient with
LVOT flow velocity $>2 \mathrm{~m} / \mathrm{s}$ ). The final sample consisted of 52 patients, aged $81.5 \pm 5.3$ years, and 28 were women (54\%). Table 1 summarizes the 2DTTE measurements for this population.

\subsection{Assessment of LVOT area and shape}

Left ventricular outflow tract diameter on 2DTTE was $2.04 \pm 0.20 \mathrm{~cm}$, while the mean LVOT diameter on 3DTEE was $2.13 \pm 0.25 \mathrm{~cm}$ and on MDCT was $2.32 \pm 0.27 \mathrm{~cm}(P<.001)$. Further findings from 3DTEE and MDCT are depicted in Table 2.

2DTTE LVOT area, estimated from the equation (LVOT diameter $/ 2)^{2} \times \Pi$, was $3.28 \pm 0.66 \mathrm{~cm}^{2}$. On the other hand, 3D imaging methods measured planimetered LVOT areas: Mean 3DTEE LVOT area was $3.95 \pm 0.90 \mathrm{~cm}^{2}$, and mean MDCT LVOT area was $4.31 \pm 0.99 \mathrm{~cm}^{2}$. The

TABLE 1 Transthoracic echocardiography data of the study sample $(n=52)$

\begin{tabular}{lc}
\hline Variable & Value \\
\hline Left ventricular ejection fraction $(\%)$ & $59.8 \pm 13.9$ \\
\hline Left ventricular diastolic volume index $\left(\mathrm{mL} / \mathrm{m}^{2}\right)$ & $58.3 \pm 18.4$ \\
\hline Left ventricular systolic volume index $\left(\mathrm{mL} / \mathrm{m}^{2}\right)$ & $25.1 \pm 14.2$ \\
\hline Stroke volume index $\left(\mathrm{mL} / \mathrm{m}^{2}\right)$ & $39.8 \pm 13.7$ \\
\hline Interventricular wall thickness $(\mathrm{cm})$ & $12.9 \pm 2.3$ \\
\hline Left ventricular posterior wall thickness $(\mathrm{cm})$ & $11.1 \pm 2.0$ \\
\hline LVOT diameter $(\mathrm{cm})$ & $2.04 \pm 0.20$ \\
\hline LVOT area (cm ${ }^{2}$ ) & $3.28 \pm 0.66$ \\
\hline Aortic valve peak velocity $(\mathrm{m} / \mathrm{s})$ & $4.4 \pm 0.6$ \\
\hline Transaortic peak systolic gradient $(\mathrm{mm} \mathrm{Hg})$ & $78.9 \pm 21.6$ \\
\hline Transaortic mean systolic gradient $(\mathrm{mm} \mathrm{Hg})$ & $49.4 \pm 14.4$ \\
\hline Aortic valve velocity-time integral $(\mathrm{cm})$ & $109.7 \pm 22.8$ \\
\hline LVOT velocity-time integral $(\mathrm{cm})$ & $20.4 \pm 6.0$ \\
\hline Dimensionless index $\left(\mathrm{LVOT}_{\mathrm{VTI}} / \mathrm{AV}{ }_{\mathrm{VTI}}\right)$ & $0.19 \pm 0.06$ \\
\hline Aortic valve area $\left(\mathrm{cm}^{2}{ }^{2}\right.$ ) by the continuity equation & $0.62 \pm 0.20$ \\
\hline Aortic valve area index $\left(\mathrm{cm}^{2} / \mathrm{m}^{2}\right.$ ) & $0.36 \pm 0.12$ \\
\hline
\end{tabular}


TABLE 2 3DTEE and MDCT imaging characteristics of the study sample $(n=52)$

\begin{tabular}{|lllc|}
\hline Variable & 3DTEE value & MDCT value & P-value \\
\hline $\begin{array}{l}\text { Maximum LVOT } \\
\text { diameter (cm) }\end{array}$ & $2.41 \pm 0.33$ & $2.83 \pm 0.33$ & $<.001$ \\
\hline $\begin{array}{l}\text { Minimum LVOT diameter } \\
(\mathrm{cm})\end{array}$ & $1.98 \pm 0.32$ & $1.93 \pm 0.27$ & .26 \\
\hline $\begin{array}{l}\text { Mean LVOT diameter } \\
(\mathrm{cm})\end{array}$ & $2.13 \pm 0.25$ & $2.35 \pm 0.27$ & $<.001$ \\
\hline $\begin{array}{l}\text { Eccentricity index } \\
\text { LVOT area }\left(\mathrm{cm}^{2}\right)\end{array}$ & $1.23 \pm 0.16$ & $1.47 \pm 0.14$ & $<.001$ \\
\hline AVA planimetry $\left(\mathrm{cm}^{2}\right)$ & $3.95 \pm 0.90$ & $4.31 \pm 0.98$ & $<.001$ \\
\hline
\end{tabular}

${ }^{*} \mathrm{n}=36$ for AVA planimetry by 3DTEE. MDCT=multidetector computed tomography; LVOT=left ventricular outflow tract

LVOT areas were all significantly different from each other $(P<.001)$. 3DTEE and MDCT LVOT areas showed very good correlation ( $r=.83$; $P<.001)$. 2DTTE LVOT area had only moderate correlation with 3DTEE LVOT area $(r=.44 ; P<.001)$ or MDCT LVOT area $(r=.48 ; P<.001)$. Compared to MDCT, 2DTTE systematically underestimates LVOT area by a mean of $1.02 \pm 0.89 \mathrm{~cm}^{2}$ (95\% confidence interval $0.78-1.27$ ) as shown by the Bland-Altman analysis (Figure 4). This difference corresponds to an underestimation of AVA by $33 \pm 30 \%$ when using the continuity equation.

The LVOT eccentricity index derived from 3DTEE (1.23 \pm 0.16$)$ was significantly different from the one derived from MDCT $(1.47 \pm 0.14)$, $P<.001$. No significant correlation was found amidst the eccentricity index and the LVOT areas obtained by each method or the differences between areas. Moreover, eccentricity was not associated with age, gender, body surface area, LVOT area, left ventricular diastolic or systolic volumes, LV ejection fraction, interventricular wall thickness, or aortic valve peak velocity (all with $\mathrm{p}=\mathrm{NS}$ ).
The intra-observer and inter-observer agreement for LVOT diameter and area measurements using the intra-class correlation coefficients were as follows: 2DTTE LVOT diameter (intra-observer 0.93 and inter-observer 0.91), 3DTEE LVOT area (intra-observer 0.96 and inter-observer 0.92), and MDCT LVOT area (intra-observer 0.97 and inter-observer 0.94).

\subsection{AVA and modified continuity equation}

2DTTE AVA estimated by the continuity equation was $0.62 \pm 0.20 \mathrm{~cm}^{2}$. Substituting the LVOT area in the continuity equation by the LVOT area acquired by 3DTEE held a mean 3DTEE fusion AVA of $0.74 \pm 0.24 \mathrm{~cm}^{2}$. When adopting the LVOT area from MDCT to the continuity equation, we obtained a mean MDCT fusion AVA of $0.80 \pm 0.24 \mathrm{~cm}^{2}$. All AVAs differed significantly among each other $(P<.001)$. 3DTEE fusion AVA showed stronger correlation with MDCT fusion AVA ( $r=.91$; $P<.001)$ than with 2DTTE AVA $(r=.75 ; P<.001)$. MDCT fusion AVA was statistically equivalent to MDCT planimetry AVA $\left(0.84 \pm 0.17 \mathrm{~cm}^{2}\right.$; $P=.12 ; r=.74$ ) (Figure 5).

Measurements of planimetric AVA by echocardiography were unattainable in several patients because of suboptimal imaging of the aortic valve opening due to heavy calcification of the cusps. We obtained planimetric AVA in 32 patients (mean AVA $0.70 \pm 0.18 \mathrm{~cm}^{2}$ ) performing 2DTTE and in 36 patients (mean AVA $0.80 \pm 0.19 \mathrm{~cm}^{2}$ ) using 3DTEE. These planimetric AVAs differed significantly between each other and from fusion AVAs $(P<.05)$.

\subsection{Patient reclassification and congruence between echocardiographic parameters and fusion AVA}

The dimensionless index (DI), derived from the ratio of $\mathrm{LVOT}_{\mathrm{VTI}} / \mathrm{AV}_{\mathrm{VTI}}$, is independent of the LVOT area measurement and is suggestive of

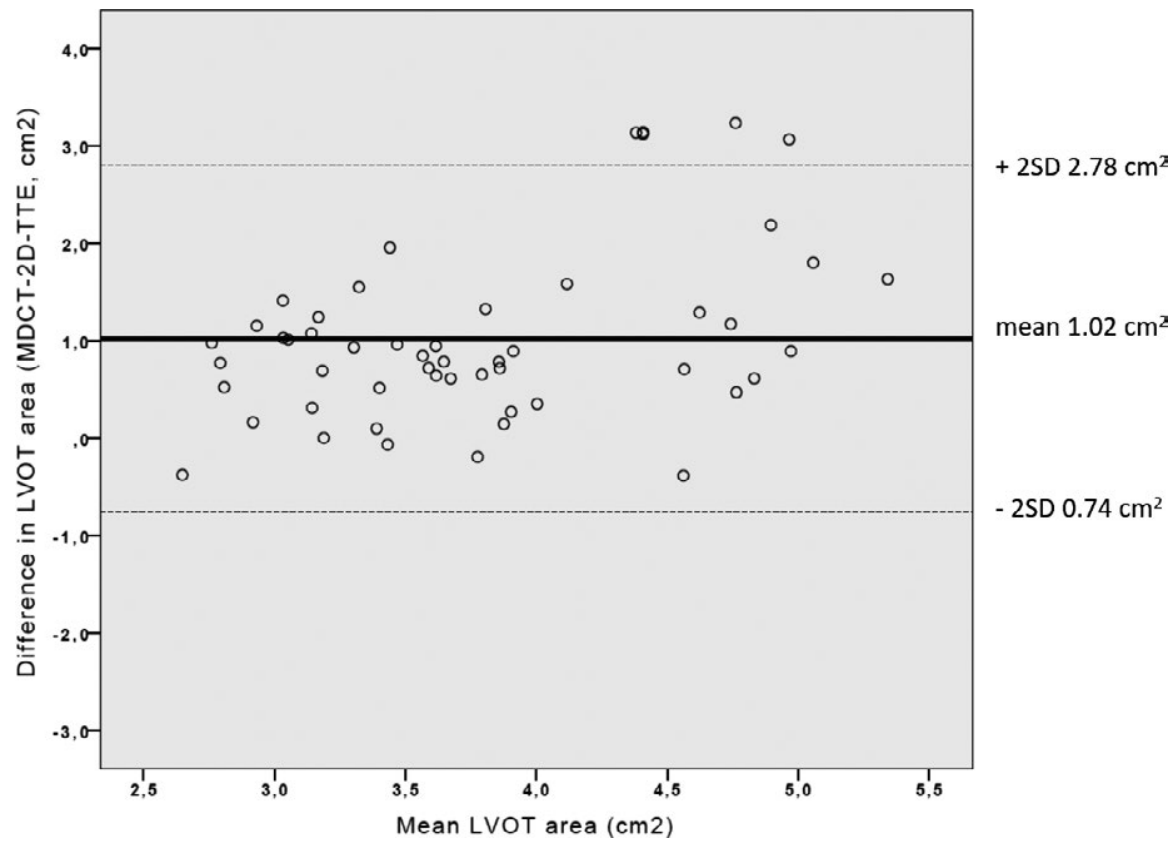

FIGURE 4 Bland-Altman plot showing the difference in LVOT area using 2DTTE versus MDCT. Solid line represents the mean difference and broken lines \pm 2 standard deviations 


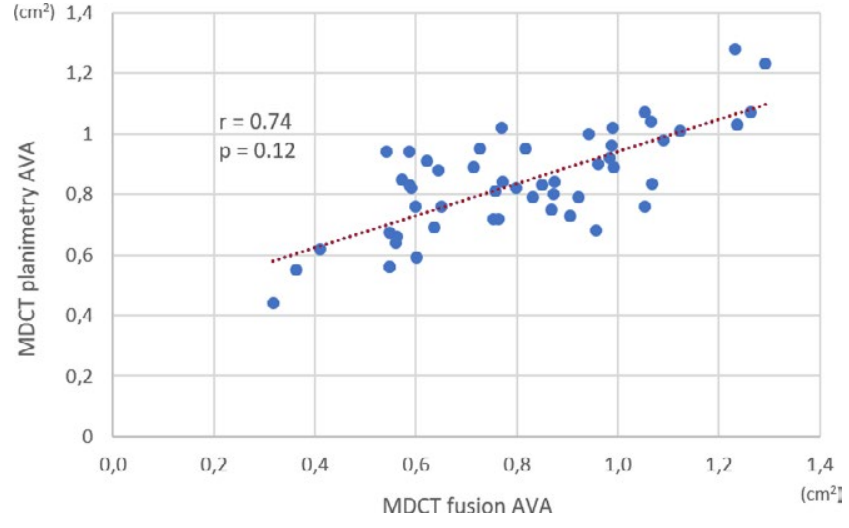

FIGURE 5 Scatter plot of correlation between MDCT fusion AVA and MDCT planimetry AVA

severe aortic stenosis when $<0.25$. In our sample, where all patients were classified as having severe aortic stenosis based on TTE AVA $<0.6 \mathrm{~cm}^{2} / \mathrm{m}^{2}$ by the continuity equation, only $79 \%$ of patients had DI $<0.25$. In contrast, when using MDCT fusion AVA the correspondence between DI $<0.25$ and AVA $<0.6 \mathrm{~cm}^{2} / \mathrm{m}^{2}$ was nearly perfect ( $98 \%$ of patients had congruence on both parameters). Furthermore, when adopting MDCT fusion AVA a total of 12 patients (24\%) would be reclassified from severe to moderate aortic stenosis. This percentage increases when analyzing the subgroup of patients with mean transaortic gradient $<40 \mathrm{~mm} \mathrm{Hg}$ despite a preserved ejection fraction, where five of nine patients were reclassified into moderate aortic stenosis. The impact of using 3DTEE fusion AVA was less pronounced with a percentage of reclassification over the whole sample of $15 \%$ (eight patients).

To improve AVA assessment by 2DTTE, we estimated a corrected 2DTTE AVA using a correction factor of 1.33, derived from the mean ratio of MDCT LVOT area/TTE LVOT area and consistent with the percentage of underestimation of AVA. The corrected TTE AVA $\left(0.83 \pm 0.27 \mathrm{~cm}^{2}\right)$ held identical values to MDCT fusion AVA $(P=.36)$ and MDCT planimetry AVA $(P=.64)$.

\section{4 | DISCUSSION}

In the present study, we found that both 2DTTE and 3DTEE underestimated LVOT area when compared to MDCT and therefore AVA estimation by echocardiography alone was significantly undervalued. Incorporating the planimetric area of the LVOT measured by MDCT into a modified multimodality continuity equation yielded significantly larger AVA values, with nearly one in four patients being reclassified from severe to moderate AS.

Moreover, we confirmed the LVOT shape is rather oval than round, which is supported by the mean eccentricity index obtained by MDCT $(1.46 \pm 0.14)$ and is in accordance with the findings of previous studies. ${ }^{6-12}$ MDCT allows planimetric measurement of the LVOT area and is therefore independent of geometric assumptions. In contrast, 2DTTE assumes a circular LVOT and relies on a single sagittal diameter mensuration which, in the setting of an eccentric LVOT, may more often correspond to the minimum diameter than to the mean diameter of the ellipse. Furthermore, the squaring of that diameter to derive the area [(LVOT diameter $\left./ 2)^{2} \times \Pi\right]$ exponentiates even small errors.

Our findings have shown that replacing 2DTTE LVOT area in the continuity equation by MDCT LVOT area resulted in a mean increase of $0.18 \mathrm{~cm}^{2}$ in AVA values. Yet performing an MDCT scan to assess the LVOT area would be impractical for most patients. 3D echocardiography which also provides threedimensional imaging of the LVOT could dismiss the need for an MDCT scan. However, previous publications have suggested that 3D transthoracic echocardiography also underestimates LVOT area when compared to MDCT. ${ }^{9-11}$ Insufficient spatial resolution, especially lateral resolution, to accurately delineate the LVOT borders has been appointed as the primary reason for the underestimation. ${ }^{9}$ The current study sought to overcome the image quality issue using TEE, routinely performed in our center for pre-TAVR assessment.

Despite improved echocardiographic window, our results showed that 3DTEE still underestimates LVOT area by a mean of $0.36 \mathrm{~cm}^{2}$ when compared to MDCT. When applying the modified continuity equation, this resulted in a mean difference of $0.06 \mathrm{~cm}^{2}$ between 3DTEE fusion AVA and MDCT fusion AVA. Although this difference is significant, 3DTEE fusion AVA correlates much strongly with MDCT fusion AVA $(r=.91)$ than with 2DTTE AVA $(r=.75)$. It remains unclear the reason for the discrepancy in the LVOT area measurements among the 3D dimensional methods. The mean LVOT eccentricity index derived from 3DTEE is inferior to the one obtained by MDCT, which could suggest 3DTEE fails to recognize more elliptical LVOTs. However, we could not establish any significant correlation between the difference of the areas and the eccentricity index or other factors. Preceding studies have also been unable to predict LVOT eccentricity or to establish a relation between it and discrepancies in the LVOT area measurements. ${ }^{9,12}$

Multimodality imaging is playing an increasingly more important role in the setting of patients with moderate-to-severe AS and prospective aortic valve replacement treatment. ${ }^{11,20-22}$ Following the results of the current and previous research, it appears that in patients with discordant calculated AVA, transaortic gradients and DI, a precise assessment of the LVOT area by MDCT, may be useful to accurately classify the severity of the AS. ${ }^{11,20,23}$ In the present study, MDCT fusion AVA was similar to MDCT planimetry AVA and MDCT fusion AVA $<0.6 \mathrm{~cm}^{2} / \mathrm{m}^{2}$ held perfect congruence with $\mathrm{DI}<0.25$, whereas 3DTEE fusion AVA failed to accomplish such concordance of parameters.

If the criteria of severe AS as AVA $<0.6 \mathrm{~cm}^{2} / \mathrm{m}^{2}$ was applied to patients assessed by MDCT fusion AVA, $24 \%$ of our cohort would be reclassified from severe to moderate AS. However, the cut-point value of $0.6 \mathrm{~cm}^{2} / \mathrm{m}^{2}$ was validated for patients studied exclusively by 2D echocardiography. Hence, reclassification of patients evaluated by MDCT fusion AVA according to that criteria should be discouraged. Nevertheless, once more data become available, the use of a hybrid or modified continuity equation accounting for the true area of the LVOT might become a useful tool for a more accurate assessment of patients with discrepant echocardiographic criteria for AS severity or poor acoustic windows. 
Bearing in mind that MDCT, 3DTEE or even 3DTTE may not be readily available or clinically indicated, we sought to establish a correction factor to compensate for LVOT ovality. We found that applying a correction factor of 1.33 to AVA measurements by 2DTTE would result in a corrected AVA that is identical to MDCT fusion AVA and MDCT planimetry AVA. In their study, Gaspar et al. ${ }^{9}$ proposed a correction factor of 1.17. However, only half of the study population had AS and, interestingly, their mean LVOT eccentricity index was also inferior to what we found. In the same study, they validate their findings on a small group of patients with severe AS. In this particular group, the LVOT mean eccentricity index (1.32 \pm 0.1 ) was higher and closer to what we describe. The inconsistency of values regarding correction factor and eccentricity index underlines the need for further and larger studies in this area.

Our study had important limitations. First and more critical is the absence of a true "gold standard" for AVA. The constraints of AVA estimation using cardiac catheterization and Gorlin formula are well known. ${ }^{24,25}$ The role of MDCT on LVOT assessment has been comprehensively described in recent publications, and we acknowledged MDCT as the most reliable method for LVOT area measurement. $^{7,9-11,15,16,20,23}$ Second, not all measurements in our study were subject to intra- and inter-observer agreement. Despite this, both echocardiography and MDCT data were collected by experienced operators and the excellent intraclass correlation coefficients obtained for the LVOT areas were reassuring of the overall data quality. Third, the present research focused exclusively on patients with severe AS, and, as such, the findings might not be applicable to patients with mild-to-moderate AS, in whom the ellipticity of the LVOT might be less accentuated. Fourth, the prognostic implications of fusion AVA need to be evaluated in prospective studies and a proper cut-point value for severe AS when using fusion AVA needs to be established. In a recent study, Clavel et al. ${ }^{26}$ have suggested that cut-point at a value of $1.2 \mathrm{~cm}^{2}$, but further research is needed.

\section{5 | CONCLUSIONS}

Three-dimensional imaging confirms the LVOT is elliptical in most patients, resulting in underestimation of LVOT area and hence AVA when assessed by 2DTTE. Although 3DTEE approximated its LVOT measurements to those of MDCT, it still failed to recognize larger areas. Incorporating the planimetric area of the LVOT measured by MDCT into a modified continuity equation yielded significantly larger AVA values, with $24 \%$ of the patients in our study being reclassified from severe to moderate AS. In patients with discrepant echocardiographic criteria for AS severity, multimodality imaging may be useful for a more accurate assessment.

\section{REFERENCES}

1. Baumgartner $\mathrm{H}$, Hung J, Bermejo J, et al. Echocardiographic assessment of valve stenosis: EAE/ASE recommendations for clinical practice. J Am Soc Echocardiogr. 2009;22:1-23.
2. Vahanian A, Alfieri O, Andreotti $F$, et al. Guidelines on the management of valvular heart disease (version 2012). Eur Heart J. 2012;33:2451-2496.

3. Nishimura RA, Otto CM, Bonow RO, et al. 2014 AHA/ACC guideline for the management of patients with valvular heart disease: a report of the American College of Cardiology/American Heart Association Task Force on Practice Guidelines. J Am Coll Cardiol. 2014;63:e57-e185.

4. Harrison MR, Gurley JC, Smith MD, Grayburn PA, DeMaria AN. A practical application of Doppler echocardiography for the assessment of severity of aortic stenosis. Am Heart J. 1988;115:622-628.

5. Oh JK, Taliercio CP, Holmes DR Jr, et al. Prediction of the severity of aortic stenosis by Doppler aortic valve area determination: prospective Doppler-catheterization correlation in 100 patients. J Am Coll Cardiol. 1988;11:1227-1234.

6. Doddamani S, Bello R, Friedman MA, et al. Demonstration of left ventricular outflow tract eccentricity by real time 3D echocardiography: implications for the determination of aortic valve area. Echocardiography. 2007;24:860-866.

7. Doddamani S, Grushko MJ, Makaryus AN, et al. Demonstration of left ventricular outflow tract eccentricity by 64-slice multi-detector CT. Int J Cardiovasc Imaging. 2009;25:175-181.

8. Burgstahler C, Kunze M, Loffler C, Gawaz MP, Hombach V, Merkle N. Assessment of left ventricular outflow tract geometry in non-stenotic and stenotic aortic valves by cardiovascular magnetic resonance. J Cardiovasc Magn Reson. 2006;8:825-829.

9. Gaspar T, Adawi S, Sachner R, et al. Threedimensional imaging of the left ventricular outflow tract: impact on aortic valve area estimation by the continuity equation. J Am Soc Echocardiogr. 2012;25:749-757.

10. Ng AC, Delgado V, van der Kley F, et al. Comparison of aortic root dimensions and geometries before and after transcatheter aortic valve implantation by 2- and 3-dimensional transesophageal echocardiography and multislice computed tomography. Circ Cardiovasc Imaging. 2010;3:94-102.

11. O'Brien B, Schoenhagen P, Kapadia SR, et al. Integration of 3D Imaging data in the assessment of aortic stenosis: impact on classification of disease severity. Circ Cardiovasc Imaging. 2011;4: 566-573.

12. Saitoh T, Shiota M, Izumo M, et al. Comparison of left ventricular outflow geometry and aortic valve area in patients with aortic stenosis by 2- dimensional versus 3-dimensional echocardiography. Am J Cardiol. 2012;109:1626-1631.

13. Minners J, Allgeier M, Gohlke-Baerwolf C, Kienzle RP, Neumann FJ, Jander N. Inconsistent grading of aortic valve stenosis by current guidelines: haemodynamic studies in patients with apparently normal left ventricular function. Heart. 2010;96:1463-1468.

14. Tops LF, Delgado V, van der Kley F, Bax JJ. Percutaneous aortic valve therapy: clinical experience and the role of multi-modality imaging. Heart. 2009;95:1538-1546

15. Vaquerizo B, Spaziano M, Alali J, et al. Three-dimensional echocardiography vs. Computed tomography for transcatheter aortic valve replacement sizing. Eur Heart J Cardiovasc Imaging. 2016;17:15-23.

16. De Vecchi C, Caudron J, Dubourg B, et al. Effect of the ellipsoid shape of the left ventricular outflow tract on the echocardiographic assessment of aortic valve area in aortic stenosis. J Cardiovasc Comput Tomogr. 2014;8:52-57.

17. Tops LF, Krishnan SC, Schuijf JD, Schalij MJ, Bax JJ. Noncoronary applications of cardiac multidetector row computed tomography. JACC Cardiovasc Imaging. 2008;1:94-106.

18. Feuchtner GM, Muller S, Bonatti J, et al. Sixty-four slice CT evaluation of aortic stenosis using planimetry of the aortic valve area. AJR Am J Roentgenol. 2007;189:197-203.

19. Bland JM, Altman DG. Statistical methods for assessing agreement between two methods of clinical measurement. Lancet. 1986;1:307-310. 
20. Kamperidis V, van Rosendael PJ, Katsanos S, et al. Low gradient severe aortic stenosis with preserved ejection fraction: reclassification of severity by fusion of Doppler and computed tomographic data. Eur Heart J. 2015;36:2087-2096.

21. Bloomfield GS, Gillam LD, Hahn R, et al. A practical guide to multimodality imaging of transcatheter aortic valve replacement. JACC Cardiovasc Imaging. 2012;4:441-455.

22. Ramineni R, Almomani A, Kumar A, Ahmad M. Role of multimodality imaging in transcatheter aortic valve replacement. Echocardiography. 2015;32:677-698.

23. Utsunomiya $\mathrm{H}$, Yamamoto $\mathrm{H}$, Horiguchi J, et al. Underestimation of aortic valve area in calcified aortic valve disease: effects of left ventricular ellipticity. Int J Cardiol. 2012;157:347-353.

24. Skjaerpe T, Hegrenaes L, Hatle L. Noninvasive estimation of valve area in patients with aortic stenosis by Doppler ultrasound and twodimensional echocardiography. Circulation. 1985;72:810-818.
25. Cannon SR, Richards KL, Crawford M. Hydraulic estimation of stenotic orifice area: a correction of the Gorlin formula. Circulation. 1985;71:1170-1178.

26. Clavel MA, Malouf J, Messika-Zeitoun D, Araoz P, Michelena H, Enriquez-Sarano M. Aortic valve area calculation in aortic stenosis by CT and Doppler echocardiography. JACC Cardiovasc Imaging. 2015;8:248-257.

How to cite this article: Pinto Teixeira P, Ramos R, Rio P, et al. Modified continuity equation using left ventricular outflow tract three-dimensional imaging for aortic valve area estimation. Echocardiography. 2017;34:978-985.

https://doi.org/10.1111/echo.13589 